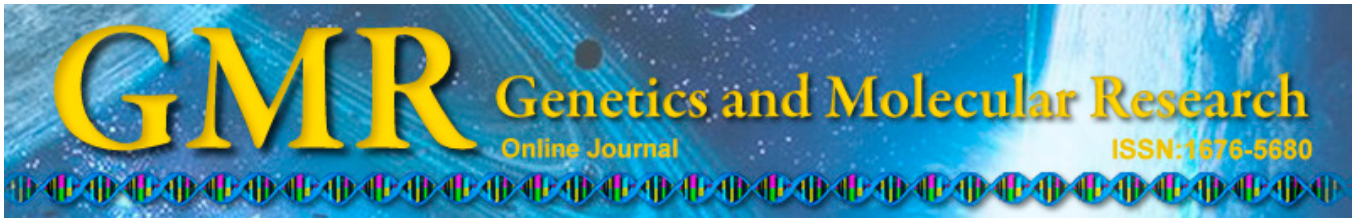

\title{
Investigation of genes in chronic and acute morphine-treated mice using microarray datasets
}

\author{
L. Ding ${ }^{1}$, J.L. Zhang ${ }^{2}$, S.H. Yu ${ }^{1}$ and L.F. Sheng ${ }^{1}$ \\ ${ }^{1}$ Department of Anesthesiology, The People's Hospital of Yinzhou, Ningbo City, \\ Zhejiang Province, China \\ ${ }^{2}$ Department of Hepatobiliary Surgery, The People's Hospital of Yinzhou, \\ Ningbo City, Zhejiang Province, China \\ Corresponding authors: S.H. Yu / L.F. Sheng \\ E-mail: shenghuiyu@yeah.net / liufangshengmed@yeah.net
}

Genet. Mol. Res. 14 (3): 10193-10205 (2015)

Received January 7, 2015

Accepted June 22, 2015

Published August 28, 2015

DOI http://dx.doi.org/10.4238/2015.August.28.3

\begin{abstract}
Morphine is a psychoactive medication that is used as a standard analgesic treatment to relieve pain in clinics. Many patients rely on chronic or acute treatment of morphine to treat pain. However, morphine is a narcotic and has a reverse effect when inappropriately used. Therefore, it is necessary to study chronic and acute morphine treatment to improve pain relief. In this study, differentially expressed genes of acute and chronic morphine-treated mice were identified using Array Express datasets. The genes that were associated with these two types of morphine treatment are discussed. A co-expression network was constructed, and the hub genes were identified. Gene ontology enrichment analysis and pathway analysis were performed using the Gene Ontology website and Kyoto Encyclopedia of Genes and Genomes, respectively. Our study revealed genes that are associated with acute and chronic morphine treatment. Therefore, this study is
\end{abstract}


potentially useful for improving pain relief of patients by acute and chronic morphine treatment.

Key words: Chronic morphine treatment; Acute morphine treatment; Gene

\section{INTRODUCTION}

Morphine is an important psychoactive chemical in opium and is used to relieve intense pain and suffering in clinical patients. It has been considered a benchmark of analgesics for its direct action on the central nervous system. Thus, many patients experiencing pain depend on chronic morphine treatment for pain relief. However, when inappropriately used, morphine has a reverse effect and causes rapidly worsening pain (Fenu et al., 2014). In recent years, many researchers have studied the influence of morphine on human biological processes. Quanhong et al. (2012) provided a novel adjuvant for morphine treatment (acute and chronic) via investigating PLCb3 factor administration in the morphine tolerance signal pathway Belkaï et al. (2013) used acute morphine treatment to study the effect of acute methadone and buprenorphine treatment on pain management and opioid addiction at the gene level. Chen et al. (2014) found that, after chronic morphine treatment, the expression of spinal G protein was upregulated. Additionally, this effect was attenuated by knockdown of the spinal mGlu5 receptor with antisense oligonucleotides. Joshi et al. (2014) evaluated the influence of morphine treatment on elevated plus maze test parameters, oxidative stress markers, and Hsp70 expression in normal and stressed rats. Consequently, morphine was shown to differentially affect acute and chronic stress-induced changes in anxiety-related behaviors and complex interactions between oxidative stress markers and Hsp 70 expression. Umathe et al. (2012) studied the differential effect of acute and chronic morphine treatment with regard to withdrawal on obsessive-compulsive behavior. The results showed that marble burying behavior was reduced after acute morphine treatment, whereas the increase of such behaviors was shown to be closely related to the withdrawal of chronic morphine treatment (Joshi et al., 2014). Although numerous studies exist on the association of acute and chronic morphine treatment with human biological processes, research on the relationship of these two basic treatment methods is scarce. In the current study, our aim was to identify differentially expressed genes (DEGs) under acute and chronic morphine treatment. We found potential functionally defined classification genes that are related to both treatments and might provide insight into how acute and chronic morphine treatment affects biological processes. We identified differential expression profiles of acute and chronic morphine treatment using the Linear Models for Microarray Data (LIMMA) Package (Smyth, 2004) in a bioconductor. Then, the differentially co-expressed genes (DCGs) were identified, and corresponding co-expression networks were constructed. After investigating the centrality characteristics of the constructed co-expression networks, functional enrichment was performed using gene ontology (GO) (Deng and Huang, 2014) and the Kyoto Encyclopedia of Genes and Genomes (KEGG) (Kanehisa et al., 2014). This research might provide a promising foundation for determining the effect of acute and chronic morphine treatment at the gene level.

\section{MATERIAL AND METHODS}

\section{Preprocess of microarray datasets}

A microarray dataset was extracted based on the transcription profile of E-GEOD-7762 
(Korostynski et al., 2007), which was obtained from Array Express. This publicly available microarray dataset was used to evaluate DEGs between 12 subjects who were acutely treated with morphine, 12 subjects who were chronically treated with morphine, and 12 normal control subjects. The dataset was processed on a Mice430_2 platform.

For data preprocess, the probe-level data were converted into expression measures. After obtaining 45,101 genes from a dataset that was read by the Affy package, the robust multiarray average method (Ma et al., 2006) and a quartile-based algorithm (Rifai and Ridker, 2001) were used to correct background and normalize the quartile data. Additionally, the MAS5 algorithm (Pepper et al., 2007), for which the value was selected via the median method, was applied to determine perfect match and mismatch values (Lacher et al., 2014). Gene screening was then carried out based on the nsFilter method of the Gene Filter package. Genes with inter-quartile ranges (Martin, 2004) greater than 0.5 were discarded. The platform annotation files that were provided by Affymetrix Company were used to map the relationship between the probes and gene symbols. A probe with no corresponding gene symbol would be filtered, and the most significant differentially expressed probe-set that was analyzed by the maximum-based method was retained if multiple probe-sets were associated with one gene. Finally, 10,313 probe genes were obtained.

\section{Identification of DEGs}

The DEGs were identified using the freely available R platform (http://cran.r-project. org/). The data were further screened by the topTable method (Smyth, 2004) in the LIMMA package, which is a Bayes method available through Bioconductor (http://www.Bioconductor. org/) (Gentleman et al., 2004). The maximum number of genes were set as 10,313, and P value was adjusted to $q$ value using the false discovery rate method (Martin, 2014). Only the genes that met our criteria $(\operatorname{logFC}>2$ and $\mathrm{P}<0.05)$ were selected as DEGs in this study.

\section{Identification of DCGs in co-expression network}

Co-expressed genes played important roles in the accomplishment of biological function and participated in similar biological pathways (Ma et al., 2014). In fact, genes with functional relationships were frequently co-expressed across the samples (Bergmann et al., 2003; Stuart et al., 2003; Lee et al., 2004).

In the current research, we used the R/EB co-express package, which uses an empirical Bayesian framework for DCG discovery (Dawson et al., 2012), to identify DCGs in mice that were treated with acute and chronic morphine compared with their corresponding normal subjects (Yang et al., 2013). The DEGs selected were applied, and the function makeMyD (Dawson, 2012) was used to convert the $\mathrm{X}$ expression matrix into the $\mathrm{D}$ correlation matrix (X refers to an m-by-n expression matrix, where rows are genes and columns are subjects; D refers to the correlation matrix output of makeMyD) by using Pearson's correlation coefficient based on a two-dimensional matrix. Then, the hub genes were calculated by rankMyGenes function (Dawson, 2012), which used a threshold to determine the names of the differently coexpressed pairs. rankMyGenes function then split those pairs into their constituent genes and created a table with the associated data. A sorted version of the table is then returned. Finally, the co-expression network was drawn by showNetwork (Dawson, 2012), a function to evaluate co-expression among a small group of genes. In the constructed network, the edges were 
colored based on the correlation strength, which was indicated by the D matrix that was input and ranged from red (strong negative correlation) to blue (strong positive correlation). The degree of the expressed correlation was set as 0.9 (Dawson, 2012).

\section{Centralities of network}

Stress was defined as the number of shortest paths passing through a node. High stress values indicated that the protein was involved in connecting regulatory molecules (Scardoni and Laudanna, 2012). Another parameter of centrality was betweenness, which described the core of node in the network. In present study, the thresholds of stress and betweeness were set as 1419.19 and 629.88 , respectively. The centralities of the networks were analyzed by the Centiscape plug-in (Scardoni et al., 2009) via mapped DEGs into the Cytoscape software (Gonçalves, 2014).

\section{Functional enrichment analysis}

To further investigate the biological functions of these genes, GO enrichment analysis and KEGG pathway enrichment analysis were performed using an online tool, the Database for Annotation, Visualization, and Integrated Discovery (DAVID) bioinformatics resource (http://david.abcc.ncifcrf.gov/tools.jsp) (Huang et al., 2008).

DAVID bioinformatics resources could systematically extract biological meaning from a large number of genes. GO terms and KEGG pathways with a P value less than 0.05 (except for cellular components in $\mathrm{GO}$ terms of chronic morphine treated, $\mathrm{P}<0.1$ ) were chosen based on expression analysis systematic explorer (EASE) test (Hosack et al., 2003), which was applied in DAVID.

EASE analysis of the regulated genes indicated molecular functions and biological processes that were unique to each category (Ford et al., 2006). The EASE score was used to detect significant categories. The threshold of EASE score, $<0.05$, and the minimum number of genes for the corresponding term, $>2$, were considered to be significant for a category.

\section{RESULTS}

\section{Identification of DEGs}

After normalization and preprocessing of the expression profile dataset, 481 and 75 DEGs of acute and chronic morphine treatment, respectively, were identified using the LIMMA package based on the research criteria $(\operatorname{logFC}>2$ and $\mathrm{P}<0.05)$. DEGs that were identified from the microarray datasets are shown in Table 1, and 23 common genes were selected, including Zbtb16, Pkp2, Plin4, Cebpa, Map3k6, Cdkn1a, Camk1g, Tsc22d3, Sgk3, Tmem125, Slc2a1, Greb11, Slc25a13, Gng11, Fkbp5, Wnt7a, Lurap11, Opalin, Arfrp1, Frmd4b, Olig2, Dusp16, and Lap3.

\section{Identification of DCGs in a co-expression network}

Twenty hub genes were identified and considered DCGs in mice treated with acute morphine. These genes included Ankrd63, A330023F24Rik, Mphosph9, Ntrk2, Sf3b1, Tbcc, 9330154F10Rik, 9630021D06Rik, Ank3, Celf2, Clk1, D4Wsu53e, Dusp8, Fam21, Gm9983, Ift20, Lonrf3, Slc7a6, Syn2, and Zfp612. A co-expression network of these 20 hub genes was drawn using the "showNetwork" function in R. However, for chronic morphine treatment DCGs, fewer hub genes were found based on empirical Bayesian algorithm. Additionally, the co-expression network was made using the top 20 DEGs that were thought to be DCGs (Figure 1). 
Genes in chronic and acute morphine-treated mice

Table 1. Identified DEGs of acute and chronic morphine treatments.

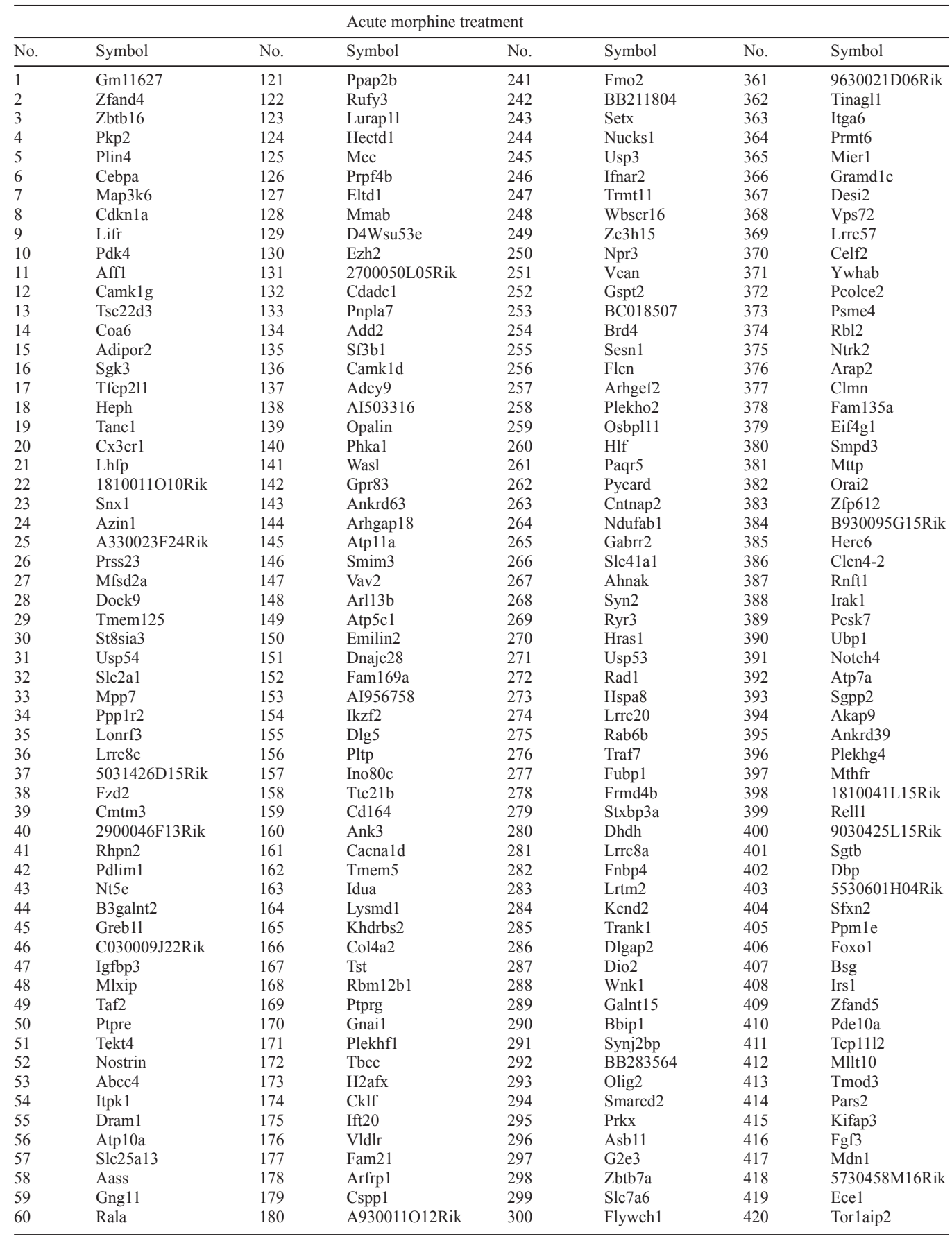

Continued on next page 
Table 1. Continued.

\begin{tabular}{|c|c|c|c|c|c|c|c|}
\hline \multirow[b]{2}{*}{ No. } & \multirow[b]{2}{*}{ Symbol } & \multirow[b]{2}{*}{ No. } & \multicolumn{5}{|c|}{ Acute morphine treatment } \\
\hline & & & Symbol & No. & Symbol & No. & Symbol \\
\hline 61 & Grm1 & 181 & Necab1 & 301 & Adamts9 & 421 & Snd1 \\
\hline 62 & Clk1 & 182 & Kcna5 & 302 & $\mathrm{~B} 830032 \mathrm{~F} 12$ & 422 & Zfand2a \\
\hline 63 & Frmpd1 & 183 & Ago3 & 303 & $\mathrm{Ssh} 2$ & 423 & 2310039F13Rik \\
\hline 64 & Tma16 & 184 & Clip1 & 304 & Dis312 & 424 & Pura \\
\hline 65 & Fzd7 & 185 & Sema5a & 305 & Cacng4 & 425 & C030023E24Rik \\
\hline 66 & Slc $8 \mathrm{a} 3$ & 186 & Rbmx & 306 & Dnajc1 & 426 & Ssr4 \\
\hline 67 & Mxd4 & 187 & Rab31 & 307 & Mphosph9 & 427 & $\mathrm{Cx} 3 \mathrm{cll}$ \\
\hline 68 & Gjb6 & 188 & Arhgef17 & 308 & Cobl & 428 & $\mathrm{Baz} 2 \mathrm{a}$ \\
\hline 69 & Osgin 2 & 189 & Crot & 309 & Gm9983 & 429 & Amy1 \\
\hline 70 & Bcl6 & 190 & Pdpk1 & 310 & Dusp16 & 430 & Fam171a2 \\
\hline 71 & Fnbp1 & 191 & Tnfrsf 25 & 311 & Sbno1 & 431 & Dact1 \\
\hline 72 & Cxxc5 & 192 & Mdm4 & 312 & Filip11 & 432 & Skp2 \\
\hline 73 & Ranbp9 & 193 & Bcr & 313 & Arhgef 28 & 433 & Ppip5k2 \\
\hline 74 & Ftx & 194 & $\mathrm{Nfe} 212$ & 314 & Nipal4 & 434 & Npas4 \\
\hline 75 & Tmem98 & 195 & Gpr22 & 315 & Fyn & 435 & Pcp411 \\
\hline 76 & Cedc6 & 196 & Atxn2 & 316 & Letmd 1 & 436 & Epha10 \\
\hline 77 & Htral & 197 & Slc6a20a & 317 & 5430440L12Rik & 437 & Gli3 \\
\hline 78 & Evalb & 198 & Ndufa 10 & 318 & Vstm5 & 438 & Scn1a \\
\hline 79 & Fn1 & 199 & Cdc3711 & 319 & Smarcc1 & 439 & $\mathrm{G} 3 \mathrm{bp} 2$ \\
\hline 80 & Ccdc141 & 200 & Cpeb3 & 320 & Lhpp & 440 & Cacna1g \\
\hline 81 & Trp53inp1 & 201 & Adam8 & 321 & Herpud1 & 441 & Tnrc18 \\
\hline 82 & Sgk1 & 202 & Zfp719 & 322 & Cntnap4 & 442 & Nacc2 \\
\hline 83 & Gpatch 8 & 203 & $\mathrm{Nr} 3 \mathrm{c} 2$ & 323 & Vip & 443 & Fam136a \\
\hline 84 & Pla2g3 & 204 & Dab2ip & 324 & Tapt1 & 444 & Primal \\
\hline 85 & Sult1a1 & 205 & Nav3 & 325 & Ankrd16 & 445 & Casp2 \\
\hline 86 & Mfsd11 & 206 & Chd4 & 326 & Ogfr & 446 & Gjc2 \\
\hline 87 & Aldh6a1 & 207 & Acot11 & 327 & D10Bwg1379e & 447 & $\mathrm{Nr} 2 \mathrm{c} 2 \mathrm{ap}$ \\
\hline 88 & Inpp5f & 208 & Ubxn7 & 328 & Nfkbiz & 448 & Nxn \\
\hline 89 & Map6d1 & 209 & Dgkg & 329 & Pld1 & 449 & Cpne7 \\
\hline 90 & Pak7 & 210 & Dusp8 & 330 & Ubtf & 450 & Ampd3 \\
\hline 91 & Ppp2r3a & 211 & Fosl2 & 331 & Chfr & 451 & Ahctfl \\
\hline 92 & Zfp410 & 212 & $\operatorname{Ccdc} 85 \mathrm{a}$ & 332 & Cxadr & 452 & $\mathrm{Nmt} 2$ \\
\hline 93 & Fkbp5 & 213 & Zfp608 & 333 & Sap30 & 453 & 6430604M11Rik \\
\hline 94 & Dpf1 & 214 & Wipf3 & 334 & Ucp2 & 454 & Wwc1 \\
\hline 95 & Tsc22d1 & 215 & Kndc1 & 335 & H2afy & 455 & Itga8 \\
\hline 96 & Spns2 & 216 & Klf4 & 336 & Per2 & 456 & $1110057 \mathrm{~K} 04 \mathrm{Rik}$ \\
\hline 97 & Pou3f1 & 217 & $\mathrm{Ccn} 12$ & 337 & Txnrd1 & 457 & $\mathrm{Sn} \times 24$ \\
\hline 98 & Csnk2a1 & 218 & Lrrc58 & 338 & Tnfrsf19 & 458 & Spred2 \\
\hline 99 & Pxdn & 219 & Zwilch & 339 & Gal3st1 & 459 & Dnajc3 \\
\hline 100 & Slc16a6 & 220 & Bicd2 & 340 & Suox & 460 & Atp6v0a2 \\
\hline 101 & Alkbh1 & 221 & Arhgef3 & 341 & P4ha2 & 461 & Fam20a \\
\hline 102 & Ctla2a & 222 & Nfat5 & 342 & Pkp4 & 462 & Magi2 \\
\hline 103 & $\mathrm{Ldb} 2$ & 223 & Acsl3 & 343 & Mat2a & 463 & Akap12 \\
\hline 104 & Tm6sfl & 224 & Pten & 344 & Fam20c & 464 & Cdk7 \\
\hline 105 & Cyp4v3 & 225 & Tmem $88 \mathrm{~b}$ & 345 & Rps6kb2 & 465 & Snw1 \\
\hline 106 & Wnt7a & 226 & Nek1 & 346 & Cacnb1 & 466 & Orc2 \\
\hline 107 & Arhgap21 & 227 & Ifrd 1 & 347 & Gm15417 & 467 & Dusp6 \\
\hline 108 & Ogt & 228 & Fgfrl1 & 348 & Prodh & 468 & Fbxl12 \\
\hline 109 & Kdsr & 229 & D1Ertd448e & 349 & Fdft 1 & 469 & Ctgf \\
\hline 110 & Gprin3 & 230 & Tm6sf2 & 350 & LOC101056642 & 470 & Klf15 \\
\hline 111 & Zfp91 & 231 & Kank3 & 351 & Slc9a2 & 471 & Fzd1 \\
\hline 112 & 9330154F10Rik & 232 & Acsbg1 & 352 & Lap3 & 472 & Tesc \\
\hline 113 & Plxdc2 & 233 & Rasal1 & 353 & P2ry 13 & 473 & Lcmt2 \\
\hline 114 & Gkn3 & 234 & Grm3 & 354 & Ppp4r1 & 474 & Snord89 \\
\hline 115 & Pnpla2 & 235 & Prpf39 & 355 & 2610011E03Rik & 475 & Gabra4 \\
\hline 116 & Mak & 236 & $\mathrm{Cp}$ & 356 & D5Wsu148e & 476 & Gm2590 \\
\hline 117 & D930009K15Rik & 237 & Sphkap & 357 & Slc1a3 & 477 & Secisbp21 \\
\hline 118 & Tnpol & 238 & 9530057J20Rik & 358 & 2700062C07Rik & 478 & 9230110C19Rik \\
\hline 119 & $\operatorname{Rin} 2$ & 239 & Ier51 & 359 & Fam $134 b$ & 479 & Bbs5 \\
\hline 120 & Plat & 240 & Mtm1 & 360 & $\operatorname{Tgm} 2$ & 480 & 2210404J11Rik \\
\hline
\end{tabular}

Continued on next page 


\begin{tabular}{|c|c|c|c|c|c|c|c|}
\hline \multirow[b]{2}{*}{ No. } & \multirow[b]{2}{*}{ Symbol } & \multirow[b]{2}{*}{ No. } & \multicolumn{5}{|c|}{ Chronic morphine treatment } \\
\hline & & & Symbol & No. & Symbol & No. & Symbol \\
\hline 1 & Zbtb16 & 20 & Arhgap36 & 39 & Neurod2 & 58 & C030034E14Rik \\
\hline 2 & Plin4 & 21 & Slc16a1 & 40 & Vat11 & 59 & Rhou \\
\hline 3 & Camk1g & 22 & Acot1 & 41 & Wscd1 & 60 & Fkbp5 \\
\hline 4 & Slc25a13 & 23 & $\mathrm{Cd} 24 \mathrm{a}$ & 42 & Sh3rf1 & 61 & Ccde106 \\
\hline 5 & Gng11 & 24 & Greb11 & 43 & Tsga10 & 62 & Tcirg1 \\
\hline 6 & Cdkn1a & 25 & Lurap11 & 44 & Lypd1 & 63 & Opalin \\
\hline 7 & Tsc $22 \mathrm{~d} 3$ & 26 & Sdk2 & 45 & Nkx2-2 & 64 & Tuba8 \\
\hline 8 & Pkp2 & 27 & Aspa & 46 & Map3k6 & 65 & Emp2 \\
\hline 9 & Rgs 10 & 28 & Kcne11 & 47 & Clspn & 66 & Tspan6 \\
\hline 10 & Ido1 & 29 & Ugt8a & 48 & Frmd4b & 67 & $\mathrm{Nr} 4 \mathrm{a} 2$ \\
\hline 11 & Map2k3 & 30 & Cebpa & 49 & Dusp16 & 68 & C030011L09Rik \\
\hline 12 & Enox 2 & 31 & Olig2 & 50 & Tmem125 & 69 & $\mathrm{Cbx} 4$ \\
\hline 13 & Dnah1 & 32 & Dusp4 & 51 & Gpr155 & 70 & Sncaip \\
\hline 14 & Sgk3 & 33 & Fabp7 & 52 & St8sia2 & 71 & Dock11 \\
\hline 15 & Wnt7a & 34 & Lap3 & 53 & Arfrp1 & 72 & AI314604 \\
\hline 16 & Rfx4 & 35 & Slc $35 d 3$ & 54 & Nexn & 73 & Lgmn \\
\hline 17 & Bhlhe40 & 36 & Mtss 1 & 55 & Ctxn2 & 74 & $1700007 \mathrm{~K} 13 \mathrm{Rik}$ \\
\hline 18 & Evi2a & 37 & BC005764 & 56 & Tef & 75 & Slc46a1 \\
\hline 19 & Ins16 & 38 & Slc2a1 & 57 & Ldlrad3 & & \\
\hline
\end{tabular}

A

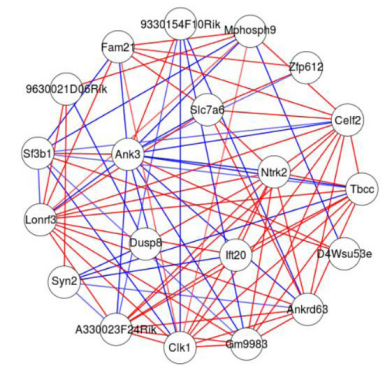

$\mathrm{B}$

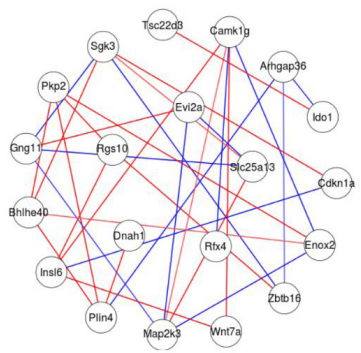

Figure1. Co-expression network of acute morphine treatment $(\mathbf{A})$ and chronic morphine treatment $(\mathbf{B})$. Nodes are genes. Edges are gene pairs, with color indicating strength of correlation, ranging from blue (negative correlation) to white (uncorrelated) and to red (positively correlated).

\section{Functional enrichment analyses}

GO analyses were carried out in three categories, including biological processes (BP), molecular functions (MF), and cellular components (CC). For GO enrichment analysis of acute morphine-treated mice, the 481 DEGs were significantly enriched in 54 BP terms, 25 
MF terms, and $18 \mathrm{CC}$ terms. Alternatively, for GO enrichment of the chronic morphine treated subject, the 75 DEGs were significantly enriched in 35 GO BP terms, 7 MF terms, and 4 CC terms. After discarding the terms with $\mathrm{P}$ values less than 0.01 , the five most significant GO $\mathrm{BP}, \mathrm{MF}$, and $\mathrm{CC}$ terms are presented in Table 2. The most significant BP, MF, and CC terms pertaining to acute morphine-treated subjects were phosphate metabolic process $(\mathrm{P}<0.01)$, Rasguanyl-nucleotide exchange factor activity $(\mathrm{P}<0.01)$, and plasma membrane $(\mathrm{P}<0.01)$, respectively. For the chronic morphine-treated subjects, the most significant corresponding $\mathrm{BP}, \mathrm{MF}$, and $\mathrm{CC}$ terms were negative regulation of cell proliferation $(\mathrm{P}<0.01)$, transcription regulator activity $(\mathrm{P}<0.05)$, and adherents junctions $(\mathrm{P}=0.06)$, respectively. The top four significant terms of GO analysis are shown in Table 2.

For KEGG pathway enrichment analysis of acute morphine-treated mice, our results showed that the DEGs were significantly enriched in eight pathways (Table 3). Alternatively, for the chronic morphine-treated subjects, the DEGs were significantly enriched in the KEGG pathway terms in cancer. These DEGs are involved in both of the acute and chronic KEGG terms.

\section{DISCUSSION}

Morphine, which is a mainstay in pain management, has been used for pain treatment for more than 5000 years (Quanhong et al., 2012). In the present study, we identified DEGs in chronic and acute morphine-treated subjects by secondary use of multiple microarray data. We then analyzed the DEGs using a network-based algorithm. The correlation between mRNA expression in chronic and acute morphine-treated subjects was characterized, and the results indicated that the common genes that correspond to DEGs might be significant.

To date, several common genes have been reported with regard to morphine treatment. $T s c 22 d 3$, which is a gene that encodes TSC22 domain family protein 3 (Vogel et al., 1996), was identified as a co-expressed gene of transcriptional activation in inbred mouse strains that were administrated acute and chronic morphine by Korostynski et al. (2007). There were differences between strains with regard to the magnitude of transcriptional response to acute morphine treatment, and the degree of gene expression tolerance relative to chronic morphine treatment was observed (Korostynski et al., 2007). Tsc22d3 has also shown to be a morphine-responsive gene for knockdown that results in alterations to dendritic spines that possibly reflects an altered potential for plastic changes in the mouse striatum (Piechota et al., 2010). Additionally, Fkbp5, which is a drug-responsive gene that codes for FKBP5, has a role in posttraumatic stress disorder, depression, and anxiety by genetic studies (Binder, 2009). Szklarczyk et al. (2012) found that traumatic stress induced the upregulation of $T s c 22 d 3$ and expression of $F k b p 5$. Moreover, traumatic stress further enhanced sensitivity to the rewarding properties of morphine.

The results of this research demonstrated that $T s c 22 d 3$ and Fkbp5 related closely with morphine treatment (Szklarczyk et al., 2012). Olig2 was identified as a novel morphine-regulated gene (Korostynski et al., 2007), because the expression of Olig2 was enhanced when the subject was exposed to morphine (Hahn et al., 2012). The hub genes of acute chronic treated subjects were also considered to have potential significance. Ntrk2, a neural differentiationand growth-associated gene, experienced dynamic changes upon morphine exposure (Wen et al., 2013). The genes mentioned above that are reported to change when exposed to morphine treatment are probably genes that are related to both chronic and acute morphine treatment. Therefore, these genes may be useful for developing novel approaches for researchers to study the impacts on biological functions in people who are treated with morphine. 
Table 2. Significant terms of GO enrichment.

\begin{tabular}{|c|c|c|}
\hline Terms & P value & Counts \\
\hline \multicolumn{3}{|l|}{ Acute morphine treatment } \\
\hline \multicolumn{3}{|l|}{$\mathrm{BP}$} \\
\hline $\begin{array}{l}\text { Phosphate } \\
\text { metabolic process }\end{array}$ & 0.0005 & $\begin{array}{l}\text { PPP2R3A, PRPF4B, NEK1, SSH2, RPS6KB2, EPHA10, CLK1, PTEN, PRKX, MTM1, PAK7, } \\
\text { MAP3K6, PDPK1, CSNK2A1, DUSP16, BRD4, IRAK1, SGK1, CAMK1G, PTPRE, BCR, } \\
\text { SGK3, PTPRG, MAK, PDK4, WNK1, CDK7, GRM1, PPM1E, FYN, NTRK2, ATP5C1, IGFBP3, } \\
\text { DUSP8, ATP6V0A2, DUSP6, CAMK1D }\end{array}$ \\
\hline $\begin{array}{l}\text { Phosphorus } \\
\text { metabolic process }\end{array}$ & 0.0005 & $\begin{array}{l}\text { PPP2R3A, PRPF4B, NEK1, SSH2, RPS6KB2, EPHA10, CLK1, PTEN, PRKX, MTM1, PAK7, } \\
\text { MAP3K6, PDPK1, CSNK2A1, DUSP16, BRD4, IRAK1, SGK1, CAMK1G, PTPRE, BCR, SGK3, } \\
\text { PTPRG, MAK, PDK4, WNK1, CDK7, GRM1, PPM1E, FYN, NTRK2, ATP5C1, IGFBP3, DUSP8, } \\
\text { ATP6V0A2, DUSP6, CAMK1D }\end{array}$ \\
\hline $\begin{array}{l}\text { Regulation of } \\
\text { cell proliferation }\end{array}$ & 0.0008 & $\begin{array}{l}\text { FOSL2, PPP2R3A, FGFRL1, FOXO1, ZBTB16, CXADR, PTEN, GLI3, ZFP91, KIFAP3, TGM2, } \\
\text { BCL6, FGF3, CEBPA, TESC, LIFR, GJB6, IRS1, PURA, HRAS1, CDKN1A, DBP, NOTCH4, MDM4, } \\
\text { IGFBP3, KLF4 }\end{array}$ \\
\hline $\begin{array}{l}\text { Regulation of } \\
\text { programmed cell death }\end{array}$ & 0.0013 & $\begin{array}{l}\text { FOXO1, ZBTB16, CX3CL1, PTEN, GLI3, PAK7, ZFP91, G2E3, TSC22D3, PYCARD, TGM2, } \\
\text { TRP53INP1, BCL6, DLG5, TRAF7, CASP2, SGK3, SKP2, HRAS1, PLEKHF1, ATP7A, CDKN1A, } \\
\text { CX3CR1, ALKBH1, IGFBP3, CAMK1D }\end{array}$ \\
\hline \multicolumn{3}{|r|}{ ( } \\
\hline Plasma membrane & 0.0059 & $\begin{array}{l}\text { SLC8A3, GPR83, SLC6A20A, FGFRL1, CXADR, SLC7A6, ARHGAP21, SLC1A3, SLC2A1, SPRED2, } \\
\text { RALA, ELTD1, DLG5, ADAM8, NT5E, AHNAK, BSG, PLD1, DAB2IP, ARHGEF2, MAGI2, CAMK1G, } \\
\text { KCND2, LIFR, TANC1, MPP7, PNPLA2, SYNJ2BP, CD164, GRM1, HRAS1, GABRR2, GRM3, PLXDC2, } \\
\text { CX3CR1, ADD2, BBS5, SCN1A, TNFRSF25, SSH2, PHKA1, CACNB1, SNX1, NOSTRIN, GNG11, EPHA10, } \\
\text { KCNA5, ZBTB16, CX3CL1, GJC2, GPR22, ECE1, PLIN4, OPALIN, SYN2, TGM2, TNFRSF19, TRAF7, } \\
\text { PRIMA1, FN1, GABRA4, DLGAP2, FRMPD1, GJB6, VAV2, ATP7A, P2RY13, FNBP1, RAB31, ADCY9, } \\
\text { ITGA6, FYN, PKP2, ITGA8, NOTCH4, PKP4, NTRK2, CACNA1G, ABCC4, STXBP3A, CP, CACNA1D, } \\
\text { ATP6V0A2 }\end{array}$ \\
\hline Cell junction & 0.0076 & $\begin{array}{l}\text { SCN1A, ARHGEF2, MAGI2, GABRA4, DLGAP2, SSH2, TANC1, MPP7, GJB6, CXADR, GJC2, GABRR2, } \\
\text { ARHGAP21, ITGA6, PKP2, PKP4, SYN2, DLG5, PRIMA1, AHNAK }\end{array}$ \\
\hline Cytoplasmic vesicle & 0.0084 & $\begin{array}{l}\text { PLAT, BSG, SGK3, YWHAB, NOSTRIN, ACSBG1, ARHGAP21, ATP7A, FNBP1, PDPK1, ECE1, SND1, } \\
\text { SLC2A1, SYN2, SPRED2, ABCC4, RAB6B, TRAF7, HSPA8, ATP6V0A2, MAP6D1 }\end{array}$ \\
\hline $\begin{array}{l}\text { Cytoplasmic membrane- } \\
\text { SYN2, }\end{array}$ & 0.0098 & PLAT, BSG, SGK3, YWHAB, NOSTRIN, ACSBG1, ATP7A, FNBP1, PDPK1, ECE1, SND1, SLC2A1, \\
\hline \multicolumn{3}{|l|}{ MF } \\
\hline $\begin{array}{l}\text { Ras guanyl-nucleotide } \\
\text { exchange factor activity }\end{array}$ & 0.0001 & PLEKHG4, ARHGEF3, ARHGEF2, BCR, WBSCR16, KIFAP3, DOCK9, ARHGEF17, VAV2, KNDC1 \\
\hline $\begin{array}{l}\text { Rho guanyl-nucleotide } \\
\text { exchange factor activity }\end{array}$ & 0.0010 & PLEKHG4, ARHGEF3, ARHGEF2, BCR, KIFAP3, DOCK9, ARHGEF17, VAV2 \\
\hline $\begin{array}{l}\text { Guanyl-nucleotide exchange } \\
\text { factor activity }\end{array}$ & 0.0016 & $\begin{array}{l}\text { PLEKHG4, ARHGEF3, ARHGEF2, BCR, WBSCR16, KIFAP3, DOCK9, ARHGEF17, D10BWG1379E, } \\
\text { VAV2, KNDC1 }\end{array}$ \\
\hline GTPase regulator activity & 0.0029 & $\begin{array}{l}\text { ARHGEF3, ARHGEF2, DAB2IP, BCR, WBSCR16, DOCK9, ARHGEF17, ARHGAP18, VAV2, ARHGAP21, } \\
\text { PLEKHG4, RASAL1, KIFAP3, RIN2, WASL, D10BWG1379E, ARAP2, KNDC1 }\end{array}$ \\
\hline \multicolumn{3}{|l|}{ Chronic morphine treatment } \\
\hline $\begin{array}{l}\text { Negative regulation of cell } \\
\text { proliferation }\end{array}$ & 0.0092 & CEBPA, CDKN1A, IDO1, ZBTB16, CD24A \\
\hline $\begin{array}{l}\text { Spinal cord oligodendrocyte } \\
\text { cell differentiation }\end{array}$ & 0.0110 & OLIG2, NKX2-2 \\
\hline $\begin{array}{l}\text { Spinal cord oligodendrocyte } \\
\text { cell fate specification }\end{array}$ & 0.0110 & OLIG2, NKX2-2 \\
\hline $\begin{array}{l}\text { Negative regulation } \\
\text { of apoptosis }\end{array}$ & 0.0114 & CDKN1A, TSC22D3, SGK3, NR4A2, IDO1 \\
\hline \multicolumn{3}{|l|}{$\mathrm{CC}$} \\
\hline Adherens junction & 0.0601 & PKP2, NEXN, RHOU \\
\hline Anchoring junction & 0.0779 & PKP2, NEXN, RHOU \\
\hline Apical part of cell & 0.0925 & TCIRG1, LGMN, SLC46A1 \\
\hline $\begin{array}{l}\text { Basolateral plasma } \\
\text { membrane }\end{array}$ & 0.0983 & SLC2A1, NEXN, RHOU \\
\hline \multicolumn{3}{|l|}{ MF } \\
\hline $\begin{array}{l}\text { Transcription regulator } \\
\text { activity }\end{array}$ & 0.0181 & CEBPA, TSC22D3, RFX4, NEUROD2, NR4A2, TEF, BHLHE40, OLIG2, ZBTB16, NKX2-2 \\
\hline $\begin{array}{l}\text { Structure-specific } \\
\text { DNA binding }\end{array}$ & 0.0273 & CLSPN, TEF, ZBTB16 \\
\hline $\begin{array}{l}\text { Protein dimerization } \\
\text { activity }\end{array}$ & 0.0275 & CEBPA, NR4A2, TEF, OLIG2, ZBTB16 \\
\hline $\begin{array}{l}\text { MAP kinase } \\
\text { phosphatase activity }\end{array}$ & 0.0399 & DUSP4, DUSP16 \\
\hline
\end{tabular}




\begin{tabular}{|c|c|c|}
\hline Terms & P value & Counts \\
\hline \multicolumn{3}{|l|}{ Acute morphine treatment } \\
\hline Pathways in cancer & 0.0002 & $\begin{array}{l}\text { CEBPA, COL4A2, BCR, SKP2, FZD1, FOXO1, ZBTB16, FZD2, PTEN, GLI3, FZD7, } \\
\text { HRAS1, CCDC6, CDKN1A, ITGA6, SLC2A1, RALA, WNT7A, FGF3, FN1 }\end{array}$ \\
\hline Melanogenesis & 0.0085 & ADCY9, GNAI1, FZD1, FZD2, WNT7A, PRKX, FZD7, HRAS1 \\
\hline MAPK signaling pathway & 0.0086 & $\begin{array}{l}\text { CACNB1, CACNG4, PRKX, HRAS1, MAP3K6, DUSP16, NTRK2, CACNA1G, } \\
\text { PLA2G3, DUSP8, CACNA1D, HSPA8, FGF3, DUSP6 }\end{array}$ \\
\hline Sphingolipid metabolism & 0.0160 & SGPP2, KDSR, PPAP2B, SMPD3, GAL3ST1 \\
\hline Dilated cardiomyopathy & 0.0201 & ITGA6, ADCY9, ITGA8, CACNB1, CACNG4, CACNA1D, PRKX \\
\hline $\begin{array}{l}\text { Arrhythmogenic right ventricular } \\
\text { cardiomyopathy (ARVC) }\end{array}$ & 0.0302 & ITGA6, PKP2, ITGA8, CACNB1, CACNG4, CACNA1D \\
\hline Basal cell carcinoma & 0.0388 & FZD1, FZD2, GLI3, WNT7A, FZD7 \\
\hline Focal adhesion & 0.0413 & PAK7, PDPK1, COL4A2, ITGA6, FYN, ITGA8, VAV2, PTEN, FN1, HRAS1 \\
\hline \multicolumn{3}{|l|}{ Chronic morphine treatment } \\
\hline Pathways in cancer & 0.0373 & CEBPA, CDKN1A, SLC2A1, ZBTB16, WNT7A \\
\hline
\end{tabular}

GO addresses the obstacle of genomic database interoperability and produces a novel, dynamic, and controlled vocabulary that can be applied to all eukaryotes. Three unique ontologies constructed on the internet (http://www.geneontology.org) are BP, MF, and CC. Among them, BP refers to genes or gene products that alter biological processes by involving a chemical or physical transformation and is accomplished by one or more ordered MF assemblies (Ashburner et al., 2000). A total of 16 BP terms (Table 4) are involved in both of acute and chronic subjects, including phosphate metabolic process, phosphorus metabolic process, regulation of cell proliferation, and regulation of programmed cell death. The most significant term for the chronic subjects was negative regulation of cell proliferation, which had a $\mathrm{P}$ value $<$ 0.01 , was also significant for the acute subjects, which had a $\mathrm{P}$ value $<0.01$. Regarding MF, three terms including protein dimerization activity $(\mathrm{P}<0.01)$, MAP kinase phosphatase activity $(\mathrm{P}<0.05)$, and MAP kinase tyrosine/serine/threonine phosphatase activity $(\mathrm{P}<0.05)$ were significant for both the acute and chronic morphine-treated subjects.

Table 4. Common GO terms of acute and chronic morphine treatment.

\begin{tabular}{|c|c|c|}
\hline Terms & $P$ value & Counts \\
\hline \multicolumn{3}{|r|}{ - 2} \\
\hline $\begin{array}{l}\text { Regulation of programmed } \\
\text { cell death }\end{array}$ & 0.0013 & $\begin{array}{l}\text { FOXO1, ZBTB16, CX3CL1, PTEN, GLI3, PAK7, ZFP91, G2E3, TSC22D3, PYCARD, } \\
\text { TGM2, TRP53INP1, BCL6, DLG5, TRAF7, CASP2, SGK3, SKP2, HRAS1, PLEKHF1, } \\
\text { ATP7A, CDKN1A, CX3CR1, ALKBH1, IGFBP3, CAMK1D }\end{array}$ \\
\hline Regulation of cell death & 0.0015 & $\begin{array}{l}\text { FOXO1, ZBTB16, CX3CL1, PTEN, GLI3, PAK7, ZFP91, G2E3, TSC22D3, PYCARD, } \\
\text { TGM2, TRP53INP1, BCL6, DLG5, TRAF7, CASP2, SGK3, SKP2, HRAS1, PLEKHF1, } \\
\text { ATP7A, CDKN1A, CX3CR1, ALKBH1, IGFBP3, CAMK1D }\end{array}$ \\
\hline $\begin{array}{l}\text { Negative regulation of cell } \\
\text { proliferation }\end{array}$ & 0.0022 & $\begin{array}{l}\text { CEBPA, TESC, PPP2R3A, FGFRL1, GJB6, ZBTB16, CXADR, GLI3, PTEN, CDKN1A, } \\
\text { KIFAP3, BCL6, IGFBP3, KLF4 }\end{array}$ \\
\hline Regulation of apoptosis & 0.0024 & $\begin{array}{l}\text { FOXO1, ZBTB16, CX3CL1, PTEN, GLI3, PAK7, G2E3, TSC22D3, PYCARD, TGM2, } \\
\text { TRP53INP1, BCL6, TRAF7, DLG5, CASP2, SGK3, SKP2, HRAS1, PLEKHF1, ATP7A, } \\
\text { CDKN1A, CX3CR1, ALKBH1, IGFBP3, CAMK1D }\end{array}$ \\
\hline $\begin{array}{l}\text { Negative regulation of } \\
\text { apoptosis }\end{array}$ & 0.0099 & $\begin{array}{l}\text { SGK3, SKP2, FOXO1, CX3CL1, PTEN, HRAS1, PAK7, TSC22D3, CDKN1A, G2E3, } \\
\text { CX3CR1, BCL6, CASP2 }\end{array}$ \\
\hline $\begin{array}{l}\text { Negative regulation of } \\
\text { programmed cell death }\end{array}$ & 0.0116 & $\begin{array}{l}\text { SGK3, SKP2, FOXO1, CX3CL1, PTEN, HRAS1, PAK7, TSC22D3, CDKN1A, G2E3, } \\
\text { CX3CR1, BCL6, CASP2 }\end{array}$ \\
\hline $\begin{array}{l}\text { Negative regulation of } \\
\text { cell death }\end{array}$ & 0.0119 & $\begin{array}{l}\text { SGK3, SKP2, FOXO1, CX3CL1, PTEN, HRAS1, PAK7, TSC22D3, CDKN1A, G2E3, } \\
\text { CX3CR1, BCL6, CASP2 }\end{array}$ \\
\hline $\begin{array}{l}\text { Negative regulation of } \\
\text { transcription }\end{array}$ & 0.0135 & $\begin{array}{l}\text { CEBPA, ZBTB7A, FZD1, NOSTRIN, SNW1, ZBTB16, GLI3, MXD4, PURA, SAP30, } \\
\text { PRMT6, PER2, BCL6, MDM4, OLIG2, BAZ2A, KLF4 }\end{array}$ \\
\hline Regulation of kinase activity & 0.0141 & $\begin{array}{l}\text { IRAK1, CDKN1A, DGKG, DUSP16, SPRED2, TRAF7, DNAJC3, VAV2, GRM1, } \\
\text { IRS1, VLDLR }\end{array}$ \\
\hline
\end{tabular}

Continued on next page 


\begin{tabular}{|c|c|c|}
\hline Terms & $P$ value & Counts \\
\hline $\begin{array}{l}\text { Regulation of transferase } \\
\text { activity }\end{array}$ & 0.0176 & $\begin{array}{l}\text { IRAK1, CDKN1A, DGKG, DUSP16, SPRED2, TRAF7, DNAJC3, VAV2, GRM1, } \\
\text { IRS1, VLDLR }\end{array}$ \\
\hline $\begin{array}{l}\text { Negative regulation of } \\
\text { nucleobase, nucleoside, } \\
\text { nucleotide and nucleic acid } \\
\text { metabolic process }\end{array}$ & 0.0227 & $\begin{array}{l}\text { CEBPA, ZBTB7A, FZD1, NOSTRIN, SNW1, ZBTB16, GLI3, MXD4, PURA, SAP30, } \\
\text { PRMT6, PER2, BCL6, MDM4, OLIG2, BAZ2A, KLF4 }\end{array}$ \\
\hline $\begin{array}{l}\text { Negative regulation of } \\
\text { nitrogen compound metabolic } \\
\text { process }\end{array}$ & 0.0249 & $\begin{array}{l}\text { CEBPA, ZBTB7A, FZD1, NOSTRIN, SNW1, ZBTB16, GLI3, MXD4, PURA, SAP30, } \\
\text { PRMT6, PER2, BCL6, MDM4, OLIG2, BAZ2A, KLF4 }\end{array}$ \\
\hline $\begin{array}{l}\text { Negative regulation of } \\
\text { macromolecule metabolic } \\
\text { process }\end{array}$ & 0.0269 & $\begin{array}{l}\text { CEBPA, ZBTB7A, SKP2, FZD1, NOSTRIN, SNW1, ZBTB16, GLI3, MXD4, PURA, } \\
\text { SAP30, SND1, PRMT6, PER2, BCL6, MDM4, OLIG2, IGFBP3, BAZ2A, KLF4 }\end{array}$ \\
\hline $\begin{array}{l}\text { Negative regulation of } \\
\text { macromolecule biosynthetic } \\
\text { process }\end{array}$ & 0.0349 & $\begin{array}{l}\text { CEBPA, ZBTB7A, FZD1, NOSTRIN, SNW1, ZBTB16, GLI3, MXD4, PURA, SAP30, } \\
\text { PRMT6, PER2, BCL6, MDM4, OLIG2, BAZ2A, KLF4 }\end{array}$ \\
\hline $\begin{array}{l}\text { Negative regulation of cellular } \\
\text { biosynthetic process }\end{array}$ & 0.0427 & $\begin{array}{l}\text { CEBPA, ZBTB7A, FZD1, NOSTRIN, SNW1, ZBTB16, GLI3, MXD4, PURA, SAP30, } \\
\text { PRMT6, PER2, BCL6, MDM4, OLIG2, BAZ2A, KLF4 }\end{array}$ \\
\hline $\begin{array}{l}\text { Negative regulation of } \\
\text { biosynthetic process }\end{array}$ & 0.0461 & $\begin{array}{l}\text { CEBPA, ZBTB7A, FZD1, NOSTRIN, SNW1, ZBTB16, GLI3, MXD4, PURA, SAP30, } \\
\text { PRMT6, PER2, BCL6, MDM4, OLIG2, BAZ2A, KLF4 }\end{array}$ \\
\hline \multicolumn{3}{|r|}{ (2: } \\
\hline Protein dimerization activity & 0.0091 & $\begin{array}{l}\text { CEBPA, HLF, FOSL2, KHDRBS2, PTPRE, IKZF2, ADIPOR2, NPR3, NPAS4, } \\
\text { ZBTB16, SGTB, DBP, SYN2, PYCARD, NFE2L2, OLIG2 }\end{array}$ \\
\hline $\begin{array}{l}\text { MAP kinase tyrosine/serine/ } \\
\text { threonine phosphatase activity }\end{array}$ & 0.0280 & DUSP16, DUSP8, DUSP6 \\
\hline MAP kinase phosphatase activity & 0.0280 & DUSP16, DUSP8, DUSP6 \\
\hline
\end{tabular}

These results demonstrated that several mRNA expressions in chronic morphinetreated subjects were also significant for acute morphine-treated subjects. However, the result of the present study needs more support in terms of clinical evidence, and the relationship between DCGs and morphine treatment needs further evaluation. Therefore, the results of functional enrichment that we identified may merit further attention, validation, and studies.

\section{ACKNOWLEDGMENTS}

Research received no specific grants from any funding agency in public, commercial, or not-for-profit sectors

\section{REFERENCES}

Ashburner M, Ball CA, Blake JA, Botstein D, et al. (2000). Gene ontology: tool for the unification of biology. Nat. Genet. 25: $25-29$.

Belkaï E, Crété D, Courtin C, Noble F, et al. (2013). Comparison of the transcriptional responses induced by acute morphine, methadone and buprenorphine. Eur. J. Pharmacol. 711: 10-18.

Bergmann S, Ihmels J and Barkai N (2003). Similarities and differences in genome-wide expression data of six organisms. PLoS Biol. 2: e9.

Binder EB (2009). The role of FKBP5, a co-chaperone of the glucocorticoid receptor in the pathogenesis and therapy of affective and anxiety disorders. Psychoneuroendocrinology 34: S186-S195.

Chen M, Zhang X, Xu H, Ma X, et al. (2014). Inhibitory effect of spinal mGlu(5) receptor antisense oligonucleotide on the up-regulated expression of spinal $\mathrm{G}$ protein associated with chronic morphine treatment. Eur. J. Pharmacol. 723 : 253-258.

Dawson JA (2012). Package 'EBcoexpress': EBcoexpress for Differential Co-Expression Analysis. R Package version 1.12.0. Available at [http://www.biocondutor.org/packages/release/bioc/manuals/EBcoexpress/man/EBcoexpress. pdf]. 
Dawson JA, Ye S and Kendziorski C (2012). R/EBcoexpress: an empirical Bayesian framework for discovering differential co-expression. Bioinformatics 28: 1939-1940.

Deng SP and Huang DS. (2014). An integrated strategy for functional analysis of microbial communities based on gene ontology and 16S rRNA gene. Int. J. Data Min. Bioinform. (in press).

Fenu S, Espa E, Cadoni C and Di CG. (2014). Conditioned saccharin avoidance induced by infusion of amphetamine in the nucleus accumbens shell and morphine in the ventral tegmental area: behavioral and biochemical study. Behav. Brain Res. 269: 55-60.

Ford G, Xu Z, Gates A, Jiang J, et al. (2006). Expression Analysis Systematic Explorer (EASE) analysis reveals differential gene expression in permanent and transient focal stroke rat models. Brain Res. 1071: 226-236.

Gentleman RC, Carey VJ, Bates DM, Bolstad B, et al. (2004). Bioconductor: open software development for computational biology and bioinformatics. Genome Biol. 5: R80.

Gonçalves E (2014). Cytocopter manual: A cytoscape plug-in for training logic models, based on CellNOptR. Available at [www.cellnopt.org/cytocopter/resources/CytocopterManual.pdf].

Hahn YK, Podhaizer EM, Hauser KF and Knapp PE (2012). HIV-1 alters neural and glial progenitor cell dynamics in the central nervous system: coordinated response to opiates during maturation. Glia 60: 1871-1887.

Hosack DA, Dennis G Jr., Sherman BT, Lane HC, et al. (2003). Identifying biological themes within lists of genes with EASE. Genome Biol. 4: R70.

Huang DW, Sherman BT and Lempicki RA. (2008). Systematic and integrative analysis of large gene lists using DAVID bioinformatics resources. Nat. Protoc. 4: 44-57.

Joshi JC, Ray A and Gulati K (2014). Differential modulatory effects of morphine on acute and chronic stress induced neurobehavioral and cellular markers in rats. Eur. J. Pharmacol. 729: 17-21.

Kanehisa M, Goto S, Sato Y, Kawashima M, et al. (2014). Data, information, knowledge and principle: back to metabolism in KEGG. Nucleic Acids Res. 42: D199-D205.

Korostynski M, Piechota M, Kaminska D, Solecki W, et al. (2007). Morphine effects on striatal transcriptome in mice. Genome Biol. 8: R128.

Lacher DW, Gangiredla J, Jackson SA, Elkins CA, et al. (2014). A novel microarray design for molecular serotyping of Shiga toxin-producing Escherichia coli isolated from fresh produce. Appl. Environ. Microbiol. 80: 4677-4682.

Lee HK, Hsu AK, Sajdak J, Qin J, et al. (2004). Coexpression analysis of human genes across many microarray data sets. Genome Res. 14: 1085-1094.

Ma C, Lv Q, Cao Y, Wang Q, et al. (2014). Genes relevant with osteoarthritis by comparison gene expression profiles of synovial membrane of osteoarthritis patients at different stages. Eur. Rev. Med. Pharmacol. Sci. 18: 431-439.

Ma L, Robinson LN and Towle HC (2006). ChREBP*Mlx is the principal mediator of glucose-induced gene expression in the liver. J. Biol. Chem. 281: 28721-28730.

Martin D (2004). Instrumentation and survey networks at the ESRF. in Eigth International Workshop on Accelerator Alignment. CERN, Geneva Switzerland.

Pepper SD, Saunders EK, Edwards LE, Wilson CL, et al. (2007). The utility of MAS5 expression summary and detection call algorithms. BMC Bioinformatics 8: 273.

Piechota M, Korostynski M, Solecki W, Gieryk A, et al. (2010). The dissection of transcriptional modules regulated by various drugs of abuse in the mouse striatum. Genome Biol. 11: R48.

Quanhong Z, Ying X, Moxi C, Tao X, et al. (2012). Intrathecal PLC(beta3) oligodeoxynucleotides antisense potentiates acute morphine efficacy and attenuates chronic morphine tolerance. Brain Res. 1472: 38-44.

Rifai N and Ridker PM (2001). Proposed cardiovascular risk assessment algorithm using high-sensitivity C-reactive protein and lipid screening. Clin. Chem. 47: 28-30.

Scardoni G and Laudanna C (2012). Centralities based analysis of complex networks. New Frontiers in Graph Theory. InTech Open.

Scardoni G, Petterlini M, and Laudanna C (2009). Analyzing biological network parameters with CentiScaPe. Bioinformatics 25: 2857-2859.

Smyth GK (2004). Linear models and empirical bayes methods for assessing differential expression in microarray experiments. Stat. Appl. Genet. Mol. Biol. 3: 3.

Stuart JM, Segal E, Koller D and Kim SK (2003). A gene-coexpression network for global discovery of conserved genetic modules. Science 302: 249-255.

Szklarczyk K, Korostynski M, Golda S, Solecki W, et al. (2012). Genotype-dependent consequences of traumatic stress in four inbred mouse strains. Genes Brain Behav. 11: 977-985.

Umathe SN, Mundhada YR and Bhutada PS (2012). Differential effects of acute morphine, and chronic morphinewithdrawal on obsessive-compulsive behavior: inhibitory influence of CRF receptor antagonists on chronic morphine-withdrawal. Neuropeptides 46: 217-221.

Vogel P, Mägert HJ, Cieslak A, Adermann K, et al. (1996). hDIP - a potential transcriptional regulator related to murine TSC-22 and Drosophila shortsighted (shs) - is expressed in a large number of human tissues. Biochim. Biophys Acta. 
1309: 200-204.

Wen A, Guo A and Chen YL (2013). Mu-opioid signaling modulates biphasic expression of TrkB and IkB $\alpha$ genes and neurite outgrowth in differentiating and differentiated human neuroblastoma cells. Biochem. Biophys. Res. Commun. 432: 638-642.

Yang J, Yu H and Liu BH (2013). Using the DCGL 2.0 package. Available at [https://cran.r-project.org/web/packages/ DCGL/DCGL.pdf]. 